\title{
Behavior of Human Leukemic Progenitor Populations in Long-Term Marrow Culture*
}

\author{
C. Eaves, L. Coulombel, I. Dubé, D. Kalousek, J. Cashman, and A. Eaves
}

\section{A. Introduction}

An analysis of mechanisms that underlie abnormal hemopoiesis requires methods that allow the cell populations involved to be characterized and quantitated under conditions that can be defined, and ideally manipulated. Although such methodologies are not yet fully developed, the past 2 decades have seen major advances in several areas relevant to this need. Of major importance has been the establishment of semisolid culture systems capable of supporting the short-term clonal growth and differentiation of individual primitive human hemopoietic progenitor cells of various types. These include pluripotent progenitors [1] as well as different classes of lineage-restricted progenitors [2-4]. Also noteworthy has been the successful exploitation of various genetic markers to examine the cell of origin of the abnormal clone in rare patients who are constitutional mosaics and in whom a clonal hemopoietic neoplasm [5, 6] has subsequently developed. The fact that in many such patients the clone responsible for the disease may continue to produce some cells that are able to differentiate relatively normally has allowed some inference to be made about the cell type initially transformed. For

* Terry Fox Laboratory, B.C. Cancer Research Centre, 601 West 10th Avenue, Vancouver, B.C. V5Z 1L3, Canada, and Departments of Medical Genetics, Pathology and Medicine, University of British Columbia, Vancouver, B.C. Canada example, in some patients with acute myoblastic leukemia (AML) all of the circulating red cells or their precursors have been found at presentation to belong to the same clone as the circulating myeloid blasts, suggesting a common derivation from a neoplastic pluripotent stem cell. On the basis of such arguments it is now generally accepted that the myeloproliferative disorders as a group as well as some cases of AML and preleukemia arise from altered pluripotent stem cells.

These latter findings have focussed attention on the need for culture systems that allow the most primitive pluripotent stemcell types to be maintained for extensive periods, ideally under conditions similar to those prevailing in the marrow of the patient so that the mechanisms controlling normal and leukemic stem-cell behavior in vivo might be accessible to study. For the initial definition of these it would be anticipated that colony assay systems might be relatively unsuitable since direct cell-cell interactions are minimized and regulation of growth and differentiation is dependent on the addition of suitable diffusible factors. Of particular interest in the case of patients with clonal neoplasms is the question not only of how leukemic stem-cell proliferation is supported, but also that of how normal hemopoiesis usually comes to be suppressed. In this latter regard the anticipated inadequacy of colony assay systems has already been demonstrated, as shown by the finding that residual normal (i.e., nonclonal) progenitors from patients with chronic myelocytic leukemia (CML) or polycythemia vera (PV) can successfully 
generate colonies of mature red cells and granulocytes in vitro, even though they fail to produce detectable numbers of mature progeny in vivo $[7,8]$. The long-term marrow culture system was thus an obvious alternative to consider.

\section{B. Long-Term Human Marrow Cultures: Present State of the Art}

In long-term marrow cultures primitive hemopoietic cells are maintained for many weeks in a confluent adherent layer composed of a variety of hemopoietic and nonhemopoietic cell types (e.g., fibroblasts) of marrow origin [9-12]. Exogenous provision of specific growth factors is not required and there is some evidence that the nonhemopoietic elements of the adherent layer substitute in this role as they may do in vivo [9].

However, in spite of the now widespread success in establishing long-term cultures from mouse bone marrow, the reproducible development and characterization of analogous cultures using human marrow has only recently been achieved [10-12]. This is due in part to the recognition that longterm maintenance of hemopoiesis in human marrow cultures depends on their initiation with specimens of adequate cellularity (i.e., aspirates containing at least $2-3 \times 10^{7}$ nucleated cells $/ \mathrm{ml}$ ), the inclusion of horse serum as well as fetal calf serum in the growth medium $[10,11]$, and the retention of half of the old growth medium at each weekly medium change $[12,13]$. In addition, it was not until detailed studies of the adherent layer were undertaken that the greater tendency of the most primitive progenitor cell types to remain in this layer in the human system was appreciated [12]. In long-term mouse marrow cultures, relatively large numbers of stem cells are released into the nonadherent fraction every week from the time the cultures are first initiated $[14,15]$. As a result the accumulated output over several weeks commonly exceeds the number initially added [15]. This is not the case in human cultures.

Evidence of stem-cell turnover can, however, be found by assessment of the pro- portion of progenitors that are in S-phase at any given time. Such evidence is obtained from a comparison of the relative numbers of colonies produced from two aliquots of a culture harvest, one of which is exposed for $20 \mathrm{~min}$ at $37^{\circ} \mathrm{C}$ to high-specific-activity tritiated thymidine, the other serving as a control. Progenitors in S-phase are inactivated by this treatment and inactivation levels of up to approximately $50 \%$ can be reproducibly demonstrated in actively cycling progenitor populations [16]. In normal marrow very few of the most primitive erythropoietic [17] and pluripotent [18] progenitor cells detectable by colony assays (i.e., primitive BFU-E and CFU-G/E, respectively) are killed by this treatment, suggesting a low level of cycling activity in these compartments. In contrast, the later erythropoietic progenitor compartments in the marrow (i.e., mature BFU-E and CFU-E) and the marrow granulopoietic colony-forming progenitor compartment (CFU-C) looked at as a whole are found to have significant S-phase components [17, 19].

In long-term marrow cultures where granulocyte and macrophage production takes place continuously, the same broadly defined granulopoietic progenitor population also shows a significant S-phase component irrespective of the age of the culture or the time since the previous medium change. Erythropoiesis is blocked at a relatively early stage of erythropoietic progenitor development so that after 4 weeks CFUEs are rarely detected and mature BFU-E numbers are also too low [15] to allow cycling data to be readily obtained. Primitive BFU-Es which, like CFU-G/Es, are almost exclusively confined to the adherent layer, show a cyclic pattern of turnover. A significant S-phase component appears transiently 2 days after each medium change but is no longer detectable 7 days later. A similar pattern of regulated cycling changes in the pluripotent stem-cell compartment with continuous cycling of the granulopoietic progenitor compartment has also been found to occur in long-term mouse marrow cultures [9]. This suggests that similar control mechanisms are operative in both systems in spite of the differences in primitive progenitor output. 


\section{Long-Term CML Marrow Cultures}

For our first experiment to compare the separate behavior of normal and leukemic progenitors in long-term marrow culture, we chose a patient with Philadelphia chromosome $\left(\mathrm{Ph}^{1}\right)$-positive disease who presented with $5 \%$ normal metaphases in his initial direct marrow preparation. Cytogenetic analysis of the erythroid and granulopoietic colonies cultured from the same marrow sample confirmed the presence of detectable $\mathrm{Ph}^{1}$-negative cells in these early hemopoietic compartments. Because of his low WBC count he remained untreated. A second marrow was obtained 1 month later. Part of this specimen was used to reassess the frequency of proliferating $\mathrm{Ph}^{1}$-negative cells and $\mathrm{Ph}^{1}$-negative progenitors in his marrow at that time. The remainder was used to initiate long-term cultures. These were maintained in the usual way [12] and 4 weeks later the adherent layers were then harvested and assayed for erythropoietic, granulopoietic, and pluripotent progenitors in standard methylcellulose assays. Cytogenetic analysis of the larger colonies obtained showed that the proportion of these that were $\mathrm{Ph}^{1}$-negative had increased from $11 \%$ (before culture) to 55\% (by 4 weeks) [20]. Thus the conditions prevailing in these cultures had clearly either favored the growth of $\mathrm{Ph}^{1}$-negative cells or had selected against the initially dominant $\mathrm{Ph}^{1}$-positive population.

We have now studied another $16 \mathrm{Ph}^{1}$ positive CML patients with the same experimental protocol. Nine of these were recently diagnosed and untreated at the time when their marrows were obtained for culture. The other seven had been previously treated for various periods (ranging from 5 months to 7 years). Twelve of the 16 have given the same result as our first experiment, i.e., a switch from an initially predominantly $\mathrm{Ph}^{1}$-positive progenitor population that rapidly declined in long-term culture to a progenitor population that within 4 weeks was predominantly $\mathrm{Ph}^{1}$ negative. In long-term cultures from three of the other four patients the $\mathrm{Ph}^{1}$-positive progenitor population also underwent a precipitous decline, but $\mathrm{Ph}^{1}$-negative progenitors did not become detectable. Pre- sumably in these cases either there were no $\mathrm{Ph}^{1}$-negative stem cells present initially or the cultures obtained from these marrow aspirates were inadequate to support the maintenance of even a normal hemopoietic population.

In one patient's long-term cultures $\mathrm{Ph}^{1}$ positive progenitors did not rapidly disappear. Rather, they showed kinetics typical of their normal counterparts in control cultures [21]. This exceptional behavior was also associated with an unusually cellular adherent layer. Because of this finding and because of the results of another experiment which showed that only $\mathrm{Ph}^{1}$-negative progenitors had adhered to the bottom of the culture at the end of the 1st week of incubation [21], we formulated the following hypothesis. It seemed that the typical failure of $\mathrm{Ph}^{1}$-positive progenitors to be maintained might simply reflect the natural death of a population critically disadvantaged by a reduced or delayed ability to become part of the adherent layer during the initial development of the culture. To test this idea we added $\mathrm{Ph}^{1}$-positive marrow or peripheral blood cells (the latter containing similar or larger numbers of primitive $\mathrm{Ph}^{1}$-positive progenitors) to preestablished marrow adherent layers derived from normal marrow. These adherent feeders had been initiated with cells from a donor of the opposite sex and kept at $37^{\circ} \mathrm{C}$ (rather than $33^{\circ} \mathrm{C}$ ) with complete removal of all nonadherent cells after 3, 7, and 14 days to reduce the hemopoietic progenitor content to undetectable levels (as shown by assays of replicate adherent layers) at the time when CML cells were added. Following the addition of the $\mathrm{Ph}^{1}$-positive $\mathrm{CML}$ cells, these cultures with and without feeders were handled in the usual way. Although only a limited number of such experiments have been completed, thus far the number of hemopoietic progenitors found in the cultures initiated on preestablished feeders has been consistently higher than the number measured in the matching control cultures without feeders, regardless of the genotype of the progenitors present. However, overall this enhancing effect has appeared to be more pronounced on the $\mathrm{Ph}^{1}$-positive population with one exception, where the $\mathrm{Ph}^{1}$-negative 
cells still became dominant. In spite of the preliminary nature of these findings, they clearly indicate that the numbers of both $\mathrm{Ph}^{1}$-positive and $\mathrm{Ph}^{1}$-negative progenitors maintained in the long-term culture system can be enhanced. Whether such manipulations will be useful for analyzing factors that favor the selective growth of one genotype over the other still remains to be determined.

Finally, we have also recently addressed the question of whether the $\mathrm{Ph}^{1}$-negative progenitors usually detectable in 4-weekold long-term CML marrow cultures are neoplastic (i.e., members of the neoplastic clone). This was tested by examining the karyotype of progenitors obtained from long-term marrow cultures established from a patient with a fertile mosaic Turner syndrome and whose $\mathrm{Ph}^{1}$-positive $\mathrm{CML}$ clone was known to have arisen in the minor (10\%) 45, X lineage [5]. All colonies analyzed from this patient's 4- to 6-weekold adherent layer assays were found to be 46, XX; i.e., they were both $\mathrm{Ph}^{1}$-negative and nonclonal [22].

\section{Long-Term AML Marrow Cultures}

Our initial observations with long-term CML marrow cultures prompted us to examine other hemopoietic malignancies to establish whether failure of neoplastic progenitor maintenance might prove to be a phenomenon of more general significance. Marrow cells from 13 newly diagnosed AML patients have been used to initiate long-term cultures and the progenitors in these cultures evaluated for 6-8 weeks. The majority showed a rapid disappearance of progenitors of abnormal (blast) colonyand cluster-forming cells to undetectable levels within 4 weeks in both nonadherent and adherent fractions. Concomitantly hemopoietic progenitors capable of producing large granulocyte colonies became readily detectable, typically for the first time. Similarly primitive erythroid progenitors frequently attained higher levels than those anticipated simply from the numbers of this type of progenitor initially detectable. In two cases cytogenetic studies were also undertaken. These confirmed that the progenitors of phenotypically normal colonies detected after 4-6 weeks were cytogenetically normal and not members of the initially prevalent chromosomally abnormal population.

As found for the long-term CML marrow cultures, this was the predominant, although not exclusive, pattern exhibited by the progenitor populations present in long-term AML marrow cultures. In fact, the maintenance of abnormal (blast) colony- and cluster-forming cells was consistently observed for 6-8 weeks in two of the AML experiments. In both of these and the other two experiments, progenitors of normal granulocyte colonies were not detectable, and only the occasional erythroid progenitor was found.

\section{E. Conclusion}

The most striking finding from these studies is the apparent incompatibility of leukemic cell growth and normal hemopoiesis both in vivo and in the long-term marrow culture system. Particularly noteworthy is the rapidity with which normal hemopoietic progenitors often become detectable as the leukemic cells and their precursors disappear. This suggests that terminal maturation may be more sensitive to the suppressive effects of a large neoplastic cell population than is the maintenance of more primitive normal hemopoietic progenitor cell types. However, preliminary evidence that the long-term culture system may be manipulated to alter favorably the ability of neoplastic cells to be maintained is also of considerable importance, since it should then be possible to delineate those factors that promote or alternatively diminish clonal dominance in vivo.

Acknowledgments. This work was supported in part by the National Cancer Institute of Canada, the British Columbia Health Care and Research Foundation, and the Ligue Nationale Contre le Cancer, France, with core support from the Cancer Control Agency of British Columbia and the British Columbia Cancer Foundation. C. Eaves is a Research Associate of the National Cancer Institute of Canada and I. Dubé was a recipient of a National Cancer Institute of Canada Studentship. 
References

1. Fauser AA, Messner HA (1978) Granuloerythropoietic colonies in human bone marrow, peripheral blood, and cord blood. Blood 52: 1243-1248

2. Senn JS, McCulloch EA, Till JE (1967) Comparison of colony-forming ability of normal and leukemic human marrow in cell culture. Lancet II:597-598

3. Eaves CJ, Eaves AC (1977) Human marrow cells capable of erythropoietic differentiation in vitro: definition of three erythroid colony responses. Blood 49:855-864

4. Messner HA, Jamal N, Izaguirre C (1982) The growth of large megakaryocyte colonies from human bone marrow. J Cell Physiol [Suppl 1]:45-51

5. Fialkow PJ (1982) Cell lineages in hematopoietic neoplasia studied with glucose6-phosphate dehydrogenase cell markers. J Cell Physiol [Suppl 1]:37-43

6. Chaganti RSK, Bailey RB, Jhanwar SC, Arlin ZA, Clarkson BD (1982) Chronic myelogenous leukemia in the monosomic cell line of a fertile Turner syndrome mosaic. Cancer Genet Cytogenet 5:215-221

7. Prchal JF, Adamson JW (1978) Polycythemia vera - the in vitro response of normal and abnormal stem cell lines to erythropoietin. J Clin Invest 61:1044-1047

8. Dubé ID, Gupta CM, Kalousek DK, Eaves CJ, Eaves AC (1984) Cytogenetic studies of early myeloid progenitor compartments in $\mathrm{Ph}^{1}$-positive chronic myeloid leukaemia (CML). Br J Haematol 56:633-644

9. Dexter TM, Spooncer E, Toksoz D, Lajtha LG (1980) The role of cells and their products in the regulation of in vitro stem cell proliferation and granulocyte development. J Supramol Struct 13:513-524

10. Gartner S, Kaplan HS (1980) Long-term culture of human bone marrow cells. Proc Natl Acad Sci USA 77:4756-4759

11. Greenberg HM, Newburger PE, Parker LM, Novak T, Greenberger JS (1981) Human granulocytes generated in continuous bone marrow culture are physiologically normal. Blood 58:724-732

12. Coulombel L, Eaves AC, Eaves CJ (1983) Enzymatic treatment of long-term human marrow cultures reveals the preferential location of primitive hemopoietic progenitors in the adherent layer. Blood 62:291-297

13. Coulombel L, Eaves CJ, Dubé ID, Kalousek DK, Eaves AC (1984) Variable persistence of leukemic progenitor cells in long-term
CML and AML marrow cultures. In: Wright DG, Greenberger JS (eds) Longterm bone marrow culture. Liss, New York, pp 243-254

14. Mauch P, Greenberger JS, Botnick L, Hannon E, Hellman S (1980) Evidence for structured variation in self-renewal capacity within long-term bone marrow cultures. Proc Natl Acad Sci USA 77:2927-2930

15. Eaves C, Coulombel L, Eaves A (1983) Analysis of hemopoiesis in long-term human marrow cultures. In: Killmann S-A, Cronkite EP, Muller-Berat CN (eds) Haemopoietic Stem Cells. Munksgaard, Copenhagen, pp 287-302

16. Becker AJ, McCulloch EA, Siminovitch L, Till JE (1965) The effect of differing demands for blood cell production on DNA synthesis by hemopoietic colony-forming cells of mice. Blood 26:296-308

17. Eaves CJ, Humphries RK, Eaves AC (1979) In vitro characterization of erythroid precursor cells and the erythropoietic differentiation process. In: Stamatoyannopoulos G, Nienhuis AW (eds) Cellular and molecular regulation of hemoglobin switching. Grune and Stratton, New York, pp 251-278

18. Fauser AA, Messner HA (1979) Proliferative state of human pluripotent hemopoietic progenitors (CFU-GEMM) in normal individuals and under regenerative conditions after bone marrow transplantation. Blood 54: 1197 $-1200$

19. Morardet N, Parmentier C (1977) Description of a suicide technique in vitro for granulocytic stem cells $\left(\mathrm{CFU}_{\mathrm{c}}\right)$ by hydroxyurea on normal human bone marrow. Biomedicine 27:349-353

20. Coulombel L, Kalousek DK, Eaves CJ, Gupta CM, Eaves AC (1983) Long-term marrow culture reveals chromosomally normal hematopoietic progenitor cells in patients with Philadelphia chromosome-positive chronic myelogenous leukemia. N Engl J Med 308: 1493-1498

21. Dubé ID, Kalousek DK, Coulombel L, Gupta CM, Eaves CJ, Eaves AC (1984a) Cytogenetic studies of early myeloid progenitor compartments in $\mathrm{Ph}^{1}$-positive chronic myeloid leukemia. II. Long-term culture reveals the persistence of $\mathrm{Ph}^{1}$-negative progenitors in treated as well as newly diagnosed patients. Blood 63:1172-1177

22. Dubé ID, Arlin ZA, Kalousek DK, Eaves CJ, Eaves AC (1984b) Nonclonal hemopoietic progenitor cells detected in long-term marrow cultures from a Turner syndrome mosaic with chronic myeloid leukemia. Blood 64: $1284-1287$ 